\title{
ON THE OPTIMAL PRICING OF A HETEROGENEOUS PORTFOLIO
}

\author{
BY
}

\author{
GENNADY I. FALIN
}

\begin{abstract}
We apply simple geometrical arguments to show that well-known approaches to determine the premium in insurance contract minimize a weighted squared differences both between the individual premiums and the individual claims and between the total premiums for classes of homogeneous risks and total claims from these blocks of business.
\end{abstract}

\section{KEYWORDS}

Individual risk model, premium, optimality.

\section{INTRODUCTION}

Consider a portfolio of $n$ heterogeneous independent insurance risks. Let $X_{i}$ be claims amount for the $i$ th risk during the period under consideration, $S$ the total loss associated with the portfolio. Under some natural assumptions (the portfolio is large enough, it is not too heterogeneous and the distribution of claim size is not too skew) the distribution of random variable $\frac{S-E S}{\sqrt{\text { Var } S}}$ can be approximated by the standard Gaussian distribution function $\Phi(x)$.

Assume that the insurer charges the premium $\pi_{i}$ for the $i$ th risk and thus collects the total premium $\pi=\sum_{i=1}^{n} \pi_{i}$. The Gaussian approximation yields that in order to guarantee sufficiently small probability of ruin $R=P(S>\pi)$ the insurer have to collect the total premium equal to

$$
\pi=E S+\sqrt{\operatorname{Var} S} \cdot z_{1-R},
$$

where $z_{\alpha}$ is $\alpha$ percentile of the standard Gaussian distribution.

Equation (1) does not say anything about the individual premiums $\pi_{i}$. In order to find them we must adopt an extra principle.

Following Zaks, Frostig and Levikson (2006) we will consider two approaches to divide the amount $\pi$ into $n$ individual premiums $\pi_{1}, \ldots, \pi_{n}$ : 
1) for given ruin probability $R=P(S>\pi)$ the weighted squared difference $\sum_{i=1}^{n} \frac{1}{s_{i}} E\left(X_{i}-\pi_{i}\right)^{2}$ between the individual risks and the individual premiums $\left(s_{1}, \ldots, s_{n}\right.$ are some known positive numbers) is minimal,

2) for given difference $\sum_{i=1}^{n} \frac{1}{S_{i}} E\left(X_{i}-\pi_{i}\right)^{2}=D$ the ruin probability $P(S>\pi)$ is minimal.

In this note with the help of simple geometrical arguments we show that both minimization problems have unique solution. Besides we prove that the optimal premiums minimize a weighted squared differences both between the individual premiums and the individual claims and between the total premiums for classes of homogeneous risks and total claims from these blocks of business.

Our contribution is motivated by a recent paper by Zaks, Frostig and Levikson (2006) who investigated similar problems of optimal pricing of a heterogeneous portfolio (which can be divided into classes of homogeneous risks) with the help of algebraic methods based on general lemmas about positive define matrices.

\section{GenERAL RESUltS ABOUT RANDOM VARIABLES}

\subsection{An optimization problem}

Let $\xi_{1}, \ldots, \xi_{N}$ be random variables with finite mean values $a_{1}, \ldots, a_{N}$ and variances $\operatorname{Var} \xi_{1}, \ldots, \operatorname{Var} \xi_{N}$. We assume that the mean values and the variances are known.

We would like to replace the random variables $\xi_{1}, \ldots, \xi_{N}$ by some non-random numbers $A_{1}, \ldots, A_{N}$ in such a way that the mean weighted squared difference

$$
D \equiv \sum_{i=1}^{N} w_{i} E\left(\xi_{i}-A_{i}\right)^{2}
$$

is minimal. Here $w_{1}, \ldots, w_{N}$ are some known positive numbers (weights).

Using elementary properties of variances of random variables we can rewrite the difference $D$ as follows:

$$
\begin{aligned}
D & =\sum_{i=1}^{N} w_{i}\left(\operatorname{Var}\left(\xi_{i}-A_{i}\right)+\left(E\left(\xi_{i}-A_{i}\right)\right)^{2}\right)=\sum_{i=1}^{N} w_{i}\left(\operatorname{Var} \xi_{i}+\left(a_{i}-A_{i}\right)^{2}\right) \\
& =\sum_{i=1}^{N} w_{i} \operatorname{Var} \xi_{i}+\sum_{i=1}^{N} w_{i}\left(a_{i}-A_{i}\right)^{2} .
\end{aligned}
$$

Since $w_{i}$ and $\operatorname{Var} \xi_{i}$ are fixed, the original minimization problem is reduced to the finding minimal value of the function 


$$
f\left(A_{1}, \ldots, A_{N}\right)=\sum_{i=1}^{N} w_{i}\left(a_{i}-A_{i}\right)^{2}
$$

Clearly, the optimal solution is

$$
A_{1}^{*}=a_{1}, \ldots, A_{N}^{*}=a_{N},
$$

and the minimal value of the function (4) equals 0 . Correspondingly the minimal value of $D$ equals $\sum_{i=1}^{N} w_{i} \operatorname{Var} \xi_{i}$.

More interesting problem arises if we put some additional restrictions on the variables $A_{1}, \ldots, A_{N}$. Taking into account further applications to insurance we consider the following problem:

Problem 1. Find minimal value of the difference (2) given that relation

$$
A_{1}+\ldots+A_{N}=C
$$

where $C$ is a known constant, holds.

Due to (3) it is sufficient to find the minimal value of the function (4) on the set (5).

To solve this problem introduce new variables $x_{i}=\sqrt{w_{i}}\left(A_{i}-a_{i}\right)$, so that $A_{i}=a_{i}+\frac{1}{\sqrt{w_{i}}} x_{i}$ (a similar transformation appears in Zaks, Frostig and Levikson, 2006, in the proof of their theorem 2). Then the problem becomes:

Problem 2. Find minimal value of the function

$$
g\left(x_{1}, \ldots, x_{N}\right)=\sum_{i=1}^{N} x_{i}^{2}
$$

given that

$$
\sum_{i=1}^{N} \frac{1}{\sqrt{w_{i}}} x_{i}=C-\sum_{i=1}^{N} a_{i}
$$

The sequences $X=\left(x_{1}, \ldots, x_{N}\right)$ and $Y=\left(\frac{1}{\sqrt{w_{i}}}, \ldots, \frac{1}{\sqrt{w_{N}}}\right)$ can be thought of as vectors in $N$-dimensional Euclidian vector space $R^{N}$. Correspondingly, the sum in the left-hand side of (7) is the inner product $X \cdot Y$ of these vectors and the function $g\left(x_{1}, \ldots, x_{N}\right)$ is $\|X\|^{2}$, where

$$
\|X\|=\sqrt{x_{1}^{2}+\ldots+x_{N}^{2}}
$$

is the length of the vector $X$. 
Further analysis is based on the Schwarz inequality (see, for example, Halmos, 1974, section 64), which states that for any two vectors $X, Y \in R^{N}$

$$
|X \cdot Y| \leq\|X\| \cdot\|Y\|,
$$

and equality occurs iff $X$ and $Y$ are linearly dependent (in particular, if $Y$ is nonzero, it means that $X$ is a scalar multiple of $Y: X=t \cdot Y$ for some $t \in R$ ).

Applying this classical theorem we have:

$$
g\left(x_{1}, \ldots, x_{N}\right)=\|X\|^{2} \geq \frac{\|X \cdot Y\|^{2}}{\|Y\|^{2}}=\frac{\left(C-\sum_{i=1}^{N} a_{i}\right)^{2}}{\sum_{i=1}^{N} \frac{1}{w_{i}}} .
$$

Thus for $\left(x_{1}, \ldots, x_{N}\right)$ satisfying $(7)$

$$
\min g\left(x_{1}, \ldots, x_{N}\right) \geq \frac{\left(C-\sum_{i=1}^{N} a_{i}\right)^{2}}{\sum_{i=1}^{N} \frac{1}{w_{i}}} .
$$

Since the vector $Y=\left(\frac{1}{\sqrt{w_{i}}}, \ldots, \frac{1}{\sqrt{w_{N}}}\right)$ is nonzero, equality in (8) occurs iff there exists a constant $t$ such that

$$
x_{i}=\frac{1}{\sqrt{w_{i}}} t, i=1, \ldots, N
$$

and (7) holds.

Set of equations (9), (7) has unique solution

$$
\begin{aligned}
& t^{*}=\frac{\left(C-\sum_{i=1}^{N} a_{i}\right)}{\sum_{i=1}^{N} \frac{1}{w_{i}}}, \\
& x_{i}^{*}=\frac{1}{\sqrt{w_{i}}} t^{*}
\end{aligned}
$$

and thus the optimal solution to the original problem 1 is

$$
\begin{gathered}
A_{i}^{*}=a_{i}+\frac{1}{\sqrt{w_{i}}} x_{i}^{*}=a_{i}+\frac{1}{w_{i}} t^{*}=a_{i}+\frac{1}{w_{i}} \frac{C-\sum_{j=1}^{N} a_{j}}{\sum_{j=1}^{N} \frac{1}{w_{j}}} . \\
D_{\min }=\sum_{i=1}^{N} w_{i} \operatorname{Var}_{i}+\frac{\left(C-\sum_{j=1}^{N} a_{j}\right)^{2}}{\sum_{j=1}^{N} \frac{1}{w_{j}}} .
\end{gathered}
$$




\subsection{The dual optimization problem}

The same approach can be applied to study the dual optimization problem:

Problem 3. Find maximum of the sum $A_{1}+\ldots+A_{N}$ if the distance

$$
\sum_{i=1}^{N} w_{i} E\left(\xi_{i}-A_{i}\right)^{2}=D
$$

is given.

Note that (3) yields that the constant $D$ must be greater or equal to $\sum_{i=1}^{N} w_{i}$ $\operatorname{Var} \xi_{i}$.

As before, rewrite the $\operatorname{sum} \sum_{i=1}^{N} w_{i} E\left(\xi_{i}-A_{i}\right)^{2}$ as $\sum_{i=1}^{N} w_{i} \operatorname{Var} \xi_{i}+\sum_{i=1}^{N} w_{i}$ $\left(a_{i}-A_{i}\right)^{2}$. Then the restriction (12) becomes

$$
\sum_{i=1}^{N} w_{i}\left(a_{i}-A_{i}\right)^{2}=D^{\prime}
$$

where $D^{\prime}=D-\sum_{i=1}^{N} w_{i} \operatorname{Var}_{i} \geq 0$.

Introducing $x_{i}=\sqrt{w_{i}}\left(A_{i}-a_{i}\right)$ we reduce the problem to the following form:

Problem 4. Find maximum of the sum $\sum_{i=1}^{N} \frac{1}{\sqrt{w_{i}}} x_{i}$ if the sum

$$
\sum_{i=1}^{N} x_{i}^{2}=D^{\prime}
$$

is given.

Similar to the problem 2, this problem can be easily solved with the help of the Schwarz inequality. We have:

$$
\sum_{i=1}^{N} \frac{1}{\sqrt{w_{i}}} x_{i}=X \cdot Y \leq\|X\| \cdot\|Y\|=\sqrt{D^{\prime}} \cdot \sqrt{\sum_{i=1}^{N} \frac{1}{w_{i}}} .
$$

Thus, for $X=\left(x_{1}, \ldots, x_{N}\right)$ satisfying condition (13)

$$
\max \sum_{i=1}^{N} \frac{1}{\sqrt{w_{i}}} x_{i} \leq \sqrt{D^{\prime}} \cdot \sqrt{\sum_{i=1}^{N} \frac{1}{w_{i}}} .
$$

Equality in (14) occurs iff there exists a constant $t$ such that

$$
x_{i}=\frac{1}{\sqrt{w_{i}}} t, i=1, \ldots, N
$$


and (13) holds.

Set of equations (15), (13) has unique solution

$$
\begin{aligned}
& t^{*}=\sqrt{\frac{D^{\prime}}{\sum_{i=1}^{N} \frac{1}{w_{i}}}}, \\
& x_{i}^{*}=\frac{1}{\sqrt{w_{i}}} \cdot t^{*}=\frac{1}{\sqrt{w_{i}}} \cdot \sqrt{\frac{D^{\prime}}{\sum_{i=1}^{N} \frac{1}{w_{i}}}} .
\end{aligned}
$$

Correspondingly, for the values $A_{i}^{*}$ which give solution to the optimization problem 3 we have:

$$
A_{i}^{*}=a_{i}+\frac{1}{w_{i}} \cdot \sqrt{\frac{D^{\prime}}{\sum_{i=1}^{N} \frac{1}{w_{i}}}} .
$$

\section{APPLICATION TO THE INDIVIDUAL RISK MODEL}

In this section we will apply the above results to the problem of optimal pricing of a heterogeneous portfolio considered by Zaks, Frostig and Levikson (2006).

Consider the individual risk model:

$$
S=X_{1}+\ldots+X_{n},
$$

where $n$ is the total number of risks in a portfolio, the random variable $X_{i}$ represents the loss associated with the $i$ th risk during the period under consideration, $S$ is the total loss for the portfolio.

We assume that the random variables $X_{1}, \ldots, X_{n}$ are independent and have finite mean values $\mu_{1}, \ldots, \mu_{n}$ and variances $\sigma_{1}^{2}, \ldots, \sigma_{n}^{2}$ respectively. Thus the random variable $S$ has finite mean $\mu=\mu_{1}+\ldots+\mu_{n}$ and variance $\sigma^{2}=\sigma_{1}^{2}+\ldots$ $+\sigma_{n}^{2}$. We also assume that for $n$ large enough the distribution function of the centered and scaled total loss, $\frac{S-\mu}{\sigma}$, can be approximated with the help of the standard Gaussian distribution $\Phi(x)=\frac{1}{\sqrt{2 \pi}} \int_{-\infty}^{+\infty} e^{-\frac{t^{2}}{2}} d t$ :

$$
P\left(\frac{S-\mu}{\sigma}<x\right) \approx \Phi(x) .
$$

Let the insurer charges the premium $\pi_{i}$ for the $i$ th risk, so that the probability of "ruin" $R$ (this event means that the total amount of all claims, $S$, is greater than the total premium collected, $\pi=\sum_{i=1}^{n} \pi_{i}$ ) is given by:

$$
R=P(S>\pi) .
$$


Using the Gaussian approximation we have:

$$
R=P\left(\frac{S-\mu}{\sigma}>\frac{\pi-\mu}{\sigma}\right) \approx 1-\Phi\left(\frac{\pi-\mu}{\sigma}\right) .
$$

Assume that the insurer is ready to accept a sufficiently small risk $R$ (say, $R=$ $1 \%$ ). Then equation (17) gives the following (approximate) formula for the total premium to be collected:

$$
\pi=\mu+\sigma \cdot z_{1-R},
$$

where $z_{\alpha}$ is $\alpha$ percentile of the standard Gaussian distribution, i.e. $\Phi\left(z_{\alpha}\right)=\alpha$.

Equation (18) does not say anything about the individual premiums $\pi_{i}$. In order to find them we must adopt an extra principle.

\subsection{Minimization of the difference between risks and premiums for given ruin probability}

Consider the weighted squared difference

$$
D \equiv \sum_{i=1}^{N} \frac{1}{s_{i}} E\left(X_{i}-\pi_{i}\right)^{2}
$$

between the individual risks $X_{1}, \ldots, X_{n}$ and the individual premiums $\pi_{1}, \ldots, \pi_{n}$ $\left(s_{1}, \ldots, s_{n}\right.$ are some known positive numbers) and require that $D$ is minimal:

$$
D \equiv D\left(\pi_{1}, \ldots, \pi_{n}\right) \rightarrow \min .
$$

Applying the results of Section 2.1 with $N=n, \xi_{i}=X_{i}, a_{i}=\mu_{i}, A_{i}=\pi_{i}, w_{i}=$ $\frac{1}{s_{i}}, C=\mu+\sigma \cdot z_{1-R}$ we can guarantee that the minimization problem (19) with the restriction (18) has unique solution (see (10)):

$$
\pi_{i}^{*}=\mu_{i}+\frac{s_{i}}{\sum_{j=1}^{n} s_{j}} \cdot \sigma \cdot z_{1-R} .
$$

Now consider the problem setting studied in Zaks, Frostig and Levikson (2006). Let the portfolio can be divided into $k$ classes of homogeneous risks with identical statistical properties of claims (usually these risks belong to the same line of business). The $i$ th class consists of $n_{i}$ risks with the same mean values equal to $\mu_{i}$ and the same variances equal to $\sigma_{i}$. Then total amount of claims from the $i$ th class, $S_{i}$, has the mean value $E S_{i}=n_{i} \mu_{i}$ and the variance $\operatorname{Var} S_{i}=n_{i} \sigma_{i}^{2}$. The total amount of all claims from the entire portfolio is $S=S_{1}+\ldots+S_{k}$ and $E S=\sum_{i=1}^{k} n_{i} \mu_{i} \equiv \mu, \operatorname{Var} S=\sum_{i=1}^{k} n_{i} \sigma_{i}^{2} \equiv \sigma^{2}$. 
Due to the homogeneity of risks from the given class $i$, the insurer should charge for all risks from the $i$ th class the same premium $\pi_{i}$. Thus the total premium is $\pi=\sum_{i=1}^{k} n_{i} \pi_{i}$.

Following Zaks, Frostig and Levikson (2006) we consider the weighted squared distance

$$
D \equiv \sum_{i=1}^{k} \frac{1}{r_{i}} E\left(S_{i}-n_{i} \pi_{i}\right)^{2}
$$

between the the total claims from different lines of business, $S_{1}, \ldots, S_{k}$, and the total premiums collected from these classes, $n_{1} \pi_{1}, \ldots, n_{k} \pi_{k}\left(r_{1}, \ldots, r_{k}\right.$ are some known positive numbers) and require that $D$ is minimal:

$$
D \equiv D\left(\pi_{1}, \ldots, \pi_{k}\right) \rightarrow \min .
$$

To have the preassigned probability of ruin we require that the relation (18) holds.

Applying the results of Section 2.1 with $N=k, \xi_{i}=S_{i}, a_{i}=n_{i} \mu_{i}, A_{i}=n_{i} \pi_{i}$, $w_{i}=\frac{1}{r_{i}}, C=\mu+\sigma \cdot z_{1-R}$ we can guarantee that the minimization problem (21) with the restriction (18) has unique solution (see (10)):

$$
n_{i} \pi_{i}^{*}=n_{i} \mu_{i}+\frac{r_{i}}{\sum_{j=1}^{k} r_{j}} \cdot \sigma \cdot z_{1-R} \Leftrightarrow \pi_{i}^{*}=\mu_{i}+\frac{r_{i}}{\sum_{j=1}^{k} r_{j}} \cdot \sigma \cdot z_{1-R} .
$$

Now return to the the minimization problem (19) with the restriction (18) and take for all risks from the $i$ th class the same value of the parameter $s$ equal to $\frac{r_{i}}{n_{i}}$. Then (20) shows that the optimal solution to the minimization problem (19) coincides with the optimal solution to the minimization problem (21). Thus the same premiums minimize a weighted squared differences both between the individual premiums and the individual claims and between the total premiums for classes of homogeneous risks and total claims from these blocks of business (with different weights).

\subsection{Minimization of the ruin probability for given difference between risks and premiums}

Now consider the dual optimization problem:

Problem 5. For the individual risk model

$$
S=X_{1}+\ldots+X_{n}
$$

find minimum of the ruin probability $R=P(S>\pi)$ given that the difference 


$$
\sum_{i=1}^{n} \frac{1}{s_{i}} E\left(X_{i}-\pi_{i}\right)^{2}=D
$$

is fixed.

Since $P(S>\pi)$ decreases as $\pi$ increases, the problem consists in finding maximum value of the total premium $\pi=\pi_{1}+\ldots+\pi_{n}$.

Applying the results of Section 2.2 with $N=n, \xi_{i}=X_{i}, a_{i}=\mu_{i}, A_{i}=\pi_{i}$, $w_{i}=\frac{1}{s_{i}}$, we can guarantee that the minimization problem (5) has unique solution (see (16)):

$$
\pi_{i}^{*}=\mu_{i}+s_{i} \sqrt{\frac{D-\sum_{i=1}^{n} \frac{1}{s_{i}} \sigma_{i}^{2}}{\sum_{i=1}^{n} s_{i}}}
$$

Now again assume that the insurance portfolio can be divided into $k$ classes of homogeneous risks with identical statistical properties of claims. The $i$ th class consists of $n_{i}$ risks with the same mean values equal to $\mu_{i}$ and the same variances equal to $\sigma_{i}$. Then total amount of claims from the $i$ th class, $S_{i}$, has the mean value $E S_{i}=n_{i} \mu_{i}$ and the variance $\operatorname{Var} S_{i}=n_{i} \sigma_{i}^{2}$. The total amount of all claims from the entire portfolio is $S=S_{1}+\ldots+S_{k}$ and $E S=\sum_{i=1}^{k} n_{i} \mu_{i} \equiv \mu$, $\operatorname{Var} S=\sum_{i=1}^{k} n_{i} \sigma_{i}^{2} \equiv \sigma^{2}$.

Due to the homogeneity of risks from the given class $i$, the insurer should charge for all risks from the $i$ th class the same premium $\pi_{i}$. Thus the total premium is $\pi=\sum_{i=1}^{k} n_{i} \pi_{i}$.

Following Zaks, Frostig and Levikson (2006) we consider the following optimization problem:

Problem 6. Find minimum of the ruin probability $R=P(S>\pi)$ given that the difference

$$
\sum_{i=1}^{k} \frac{1}{r_{i}} E\left(S_{i}-n_{i} \pi_{i}\right)^{2}=D
$$

is fixed.

Since $P(S>\pi)$ decreases as $\pi$ increases, the problem consists in finding maximum value of the total premium $\pi=n_{1} \pi_{1}+\ldots+n_{k} \pi_{k}$.

Applying the results of Section 2.2 with $N=k, \xi_{i}=S_{i}, a_{i}=n_{i} \mu_{i}, A_{i}=n_{i} \pi_{i}$, $w_{i}=\frac{1}{r_{i}}$, we can guarantee that the minimization problem 6 has unique solution (see (16)):

$$
\pi_{i}^{*}=\mu_{i}+\frac{r_{i}}{n_{i}} \sqrt{\frac{D-\sum_{i=1}^{k} \frac{1}{r_{i}} n_{i} \sigma_{i}^{2}}{\sum_{i=1}^{k} r_{i}}}
$$




\section{REFERENCES}

Halmos, P.R. (1974) Finite-Dimensional Vector Spaces. Springer.

ZAKS Y., Frostig E. and Levikson B. (2006) Optimal pricing of a heterogeneous portfolio for a given risk level. ASTIN Bulletin 36(1), 161-185.

Prof. DR. GenNady Falin

Department of Probability

Mechanics and Mathematics Faculty

Moscow State University

Moscow 119992, Russian Federation

Tel.: +7 4959391403

E-mail: gennadi_falin@mtu-net.ru 